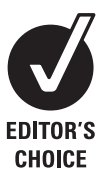

CHOICE

\title{
Cancers related to viral agents that have a direct role in carcinogenesis: pathological and diagnostic techniques
}

\author{
Antonino Carbone, ${ }^{1}$ Paolo De Paoli ${ }^{2}$
}

${ }^{1}$ Division of Pathology, Centro di Riferimento Oncologico, IRCCS, National Cancer Institute,

Aviano, Italy

${ }^{2}$ Scientific Directorate, Centro di Riferimento Oncologico, IRCCS, National Cancer Institute, Aviano, Italy

\section{Correspondence to}

Dr Paolo De Paoli, Centro di Riferimento Oncologico, IRCCS, National Cancer Institute, Via Gallini, 2, Aviano 33081, Italy: dirscienti@cro.it

\section{Search strategy and} selection criteria References for this review were identified through searches of PubMed for articles published from January 1992 to August 2010, by use of the terms vaccination, viral agents, carcinogenesis, oncogenic viruses. Articles from IARC Monographs have also been reviewed.

AC has been a member of WHO IARC Monograph Working Group on Biological Agents, Lyon-2009.

Accepted 20 February 2012

\section{ABSTRACT}

The International Agency for Research on Cancer has recently reassessed the carcinogenicity of the biological agents classified as 'carcinogenic to humans'. Among the biological agents having a direct role in carcinogenesis, Epstein-Barr virus, Kaposi's sarcomaassociated herpes virus and human papillomavirus contribute to a variety of malignancies worldwide in humans including nasopharyngeal carcinoma, several types of lymphomas, genital tract carcinomas and Kaposi's sarcoma. The authors review the current knowledge on cancers that have been attributed to Epstein-Barr virus, Kaposi's sarcoma-associated herpes virus and human papillomavirus looking at the pathological classification of these cancers and description of the implicated viruses, highlighting a wide range of pathological and virological diagnostic techniques. This review also focuses on the new oncological scenario ahead, once strategies against carcinogenic infectious agents are found to be effective.

\section{INTRODUCTION}

Approximately $15-20 \%$ of cancers worldwide have been attributed to infectious agents. The second most frequent cancer in women-cervical cancerand about $80 \%$ of liver cancers are associated with specific viral infections and are proved to be caused by such agents. The proportion of infectionattributable cancer is higher in developing countries $(26 \%)$ than in developed countries (8\%), reflecting the higher prevalence of infection with the major causative agents (eg, hepatitis B virus (HBV), Helicobacter pylori, human papillomavirus (HPV) and HIV-1), and lack of screening for HPV-related cervical precancerous lesions. ${ }^{1}$

The International Agency for Research on Cancer (IARC) has recently reassessed the carcinogenicity of the biological agents classified as 'carcinogenic to humans' (group 1), and has identified additional tumour sites and mechanisms of carcinogenesis. IARC group 1 agents assessed by the IARC monograph working group include Epstein-Barr virus (EBV), HBV, hepatitis C virus (HCV), Kaposi's sarcoma-associated herpes virus (KSHV), HIV-1, HPV-16, human T-lymphotropic virus-1 (HTLV-1), Helicobacter pylori, Clonorchis sinensis, Opistorchis viverrini and Schistosoma haematobium. ${ }^{2}$

EBV, HPV, HTLV-1 and KSHV play a direct role in carcinogenesis-encoding oncoproteins that are able to promote cellular transformation. Other infectious agents appear to have an indirect role, either inducing a chronic inflammation (HBV, HCV and Helicobacter pylori) or mediating its effects on cancer risk by lowering host immunity to other oncogenic infections (HIV-1). ${ }^{1}$

In this paper, we review the current knowledge on cancers that have been attributed to EBV, KSHV and HPV. The focus is on the classification and multidisciplinary integrative diagnosis of these cancers. The review is organised in three parts. The first is an introductory section looking at pathological classification of infection-attributable cancers with a brief description of the implicated viruses. The second section represents a wide range of pathological and virological diagnostic techniques. By reporting current knowledge, this section focuses on practical information with emphasis on clinical application. The final section, including concluding remarks, looks at the new oncological scenario that we will probably face ahead of us once strategies against carcinogenic infectious agents are found to be effective.

\section{EBV, KSHV, HPV AND ATTRIBUTED CANCERS}

Epstein-Barr virusEBV is a $\gamma$-herpesvirus that has infected $>90 \%$ of the world's population. Interhuman transmission occurs by the oral route, followed by productive oropharyngeal infection; then the virus infects circulating B lymphocytes, leading to the persistence of viral DNA as an episome, thus establishing a latent infection. ${ }^{3} 4$

Experimental data have shown substantial differences in B lymphocytes as compared with epithelial cells concerning viral gene expression, genome amplification and genome loss. ${ }^{5-7} \mathrm{~A}$ common characteristic of the virus consists of the achievement of oncogenicity after activation of intracellular signalling pathways involved in the control of proliferation.

\section{Cancers that have been attributed to EBV}

There is sufficient evidence for a causative role of EBV in nasopharyngeal carcinoma (NPC), endemic Burkitt's lymphoma (BL), immune suppressionrelated lymphomas, extranodal natural killer $\mathrm{T}$ (NK/T)-cell lymphoma (nasal type), and a subset of Hodgkin's lymphoma (HL) ${ }^{2}$ (table 1, figure 1 ).

NPC is rare in most populations around the world but common in southeast Asia. According to the WHO international histological classification of tumours, $^{8}$ NPCs are classified into three classes: keratinising carcinoma, differentiated non-keratinising carcinoma and undifferentiated carcinoma Most NPCs diagnosed in the 'high-risk' areas belong to the last two classes. ${ }^{89}$ In undifferentiated carcinoma there is a very strong association with 
Table 1 Biological agents by the International Agency for Research on Cancer monograph working group (adapted from Bouvard et al ${ }^{2}$ )

\begin{tabular}{lll}
\hline Group 1 agent & Cancers for which there is sufficient evidence in humans & Established mechanistic events \\
\hline Epstein-Barr virus (EBV) & $\begin{array}{l}\text { Nasopharyngeal carcinoma, Burkitt lymphoma, } \\
\text { immunosuppression-related non-Hodgkin's lymphoma, } \\
\text { extranodal NK/T-cell lymphoma (nasal type), Hodgkin lymphoma } \\
\text { Kaposi sarcoma herpesvirus (KSHV) }\end{array}$ & $\begin{array}{l}\text { Cell proliferation, inhibition of apoptosis, } \\
\text { Genomic instability, cell migration }\end{array}$ \\
Human papilloma virus type 16 (HPV-16) & $\begin{array}{l}\text { Carcinoma of the cervix, vulva, vagina, penis, anus, } \\
\text { oral cavity and oropharynx and tonsil }\end{array}$ & $\begin{array}{l}\text { genomic instability, cell migration } \\
\text { Immortalisation, genomic instability, inhibition } \\
\text { of DNA damage response, anti-apoptotic activity }\end{array}$
\end{tabular}

NK cells, natural killer cells.

$\mathrm{EBV}^{8} 9$ (figure 2). Carcinomas with a histological similarity to NPC called lymphoepithelial-like carcinomas, can occur in multiple organ sites and have been reported most frequently in the stomach. A recent review of over 30000 cancers of the stomach identified evidence of EBV DNA in $8 \%$ of the patients. ${ }^{10}$ EBV DNA is present within tumour cells and not in the surrounding epithelium. ${ }^{10}$

Endemic BL is a highly proliferative B-cell tumour that includes three variants: endemic (affecting children in equatorial Africa and New Guinea), sporadic (affecting children and young adults all over the world), and immunodeficiency related (primarily in association with HIV infection). EBV has been proved to be present in all cases of the endemic variant, 15-20\% of cases of the sporadic variant, and $30-40 \%$ of cases of the immunodeficiency-related variant. ${ }^{11}$

EBV-associated lymphomas in the immunosuppressed AIDS patients include BL, diffuse large B-cell lymphoma with immunoblastic morphology, primary central nervous system lymphoma, KSHV positive or EBV positive primary effusion lymphoma (PEL) (figure 1), and plasmablastic lymphoma of the oral cavity type (for a recent review, see Carbone et al ${ }^{12}$ ). The proportion of tumours associated with EBV ranges between $40 \%$ and $100 \%$ and depends on the histological type.

Lymphoproliferations, referred to as post-transplant lymphoproliferative disorders, and classified histologically as diffuse large B-cell lymphoma, have been considered as EBV-driven lymphoproliferations occurring in the context of defective T-cell immunity against EBV. ${ }^{13}$

EBV is an established cause of extranodal NK/T-cell lymphoma (nasal type), previously called angiocentric $\mathrm{T}$-cell lymphoma. ${ }^{2}$ Several recent large case series confirm the presence of EBV in tumour cells in nearly $100 \%$ of cases. Nasal NK/T-cell lymphoma cells exhibit several unique features including an absence of T-cell antigens and the expression of the NK cell marker CD56. ${ }^{11}$

Other T-cell lymphoproliferative disorders that have been reported to be associated with EBV include a subset of peripheral
T-cell lymphomas, enteropathy-type T-cell lymphomas, $\gamma \delta$

T-cell lymphomas (hepatosplenic and non-hepatosplenic), T-cell lymphoproliferative disorders after chronic EBV infection, EBV-associated cutaneous T-cell lymphoproliferative disorders (especially in Asia) and aggressive NK-cell leukaemias/ lymphomas.

Angioimmunoblastic T-cell lymphoma is a distinct entity of peripheral T-cell lymphoma, which is also nearly always associated with EBV. Angioimmunoblastic T-cell lymphoma is characterised by prominent proliferation of high endothelial venules and follicular dendritic cells. ${ }^{11}$

Not all subtypes of classic HL (cHL) harbour EBV to the same degree. There are also data that suggest that the incidence of EBV-positive cHL is age-related.

Based on patterns of expression of the EBV genome, three types of latent gene expression have been reported: latency I, II and III. ${ }^{13}$ Whole gene expression and associated malignancies are reported in table 2 .

In AIDS-associated diffuse large B-cell lymphoma, viral gene expression patterns are variable, but the transforming EBV LMP1 is frequently expressed. ${ }^{13} 14$

In BL, EBV adopts the 'latency I' pattern, which involves only the expression of EBNA-1 and EBERs. The absence of EBV from the majority of sporadic or AIDS-associated BL indicates that it is not essential in the pathogenesis of this tumour. Conversely, the key pathogenic event in $\mathrm{BL}$ is the $\mathrm{Ig} / \mathrm{c}-$ myc translocation found in all $\mathrm{BL}^{3}{ }^{15}$

In AIDS patients, HL is frequently associated with EBV infection (90-100\% of cases) and can be considered as an EBVdriven lymphoma in which EBV-encoded LMP1 is thought to play a pivotal pathogenetic role. In these cases, in fact, the Hodgkin Reed-Sternberg cells, the neoplastic component of HL tumour, show consistent evidence of the EBV 'latency II' pattern, which involves expression of the transforming EBV protein LMP1, and EBNA-1 and LMP2A. ${ }^{12}$ 16-19

In many PEL cases, the lymphoma cells harbour both EBV and KSHV, while some are only positive for KSHV. ${ }^{12}$ This event and
Figure 1 Relationship of HIVassociated lymphomas with Epstein-Barr virus and Kaposi's sarcoma-associated herpes virus/HHV8associated lymphoproliferative disorders. Reprinted with permission from Carbone A, Cesarman E, Spina M, et al. HIV-associated lymphomas and gamma-herpesviruses. Blood 2009; 113:1213-24. ${ }^{12}$ (C)The American Society of Haematology. BL, Burkitt's lymphoma; DLBCL, diffuse large B-cell lymphoma; EBV, Epstein-Barr virus; HHV-8, human herpes virus 8; KSHV, Kaposi's sarcoma-associated herpes virus; MCD, multicentric Castleman's disease; PEL, primary effusion lymphoma.

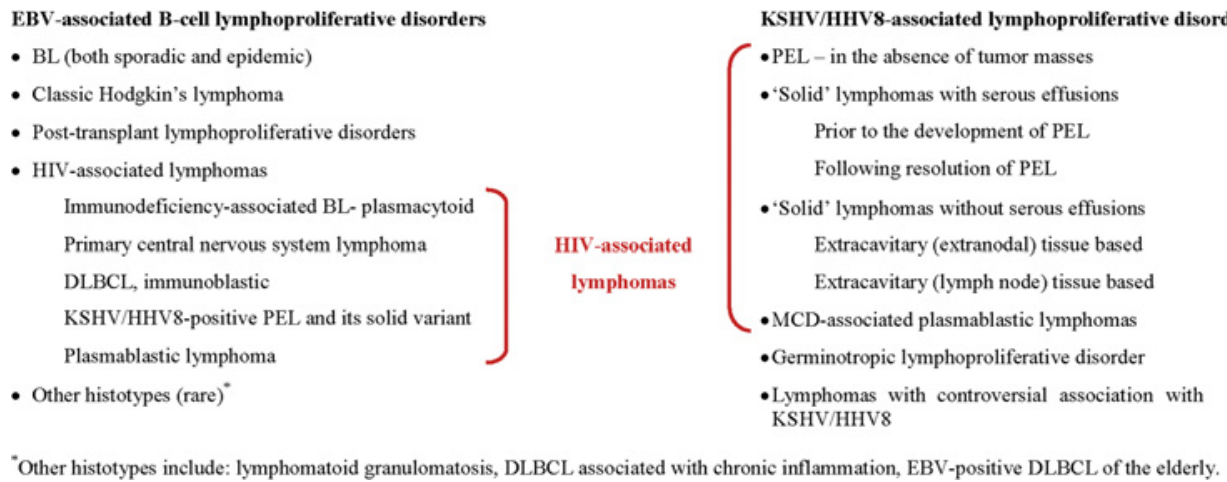

(1)

$$
\text { KSHV/HHV8 }
$$

Other histotypes include: lymphomatoid granulomatosis, DLBCL associated with chronic inflammation, EBV-positive DLBCL of the elderly. 


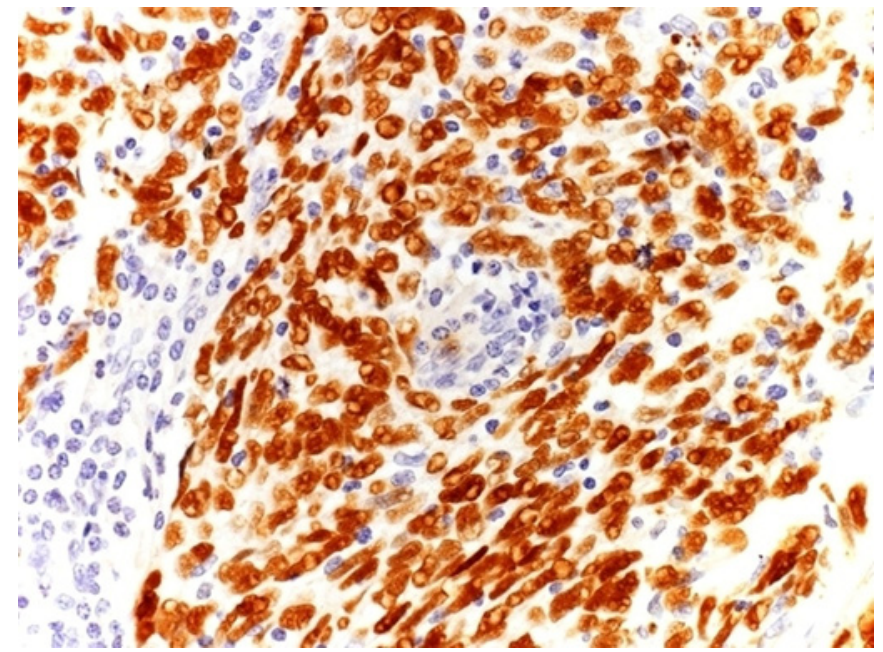

Figure 2 Epstein-Barr virus (EBV)-associated nasopharyngeal carcinoma. Almost all carcinoma cells exhibit nuclear positivity for EBV with a strong staining intensity. EBV positivity assessed by EBER-in situ hybridisation in formalin-fixed paraffin-embedded tissue section. Haematoxylin counterstain, original magnification $\times 40$.

the fact that the (non-transforming) EBV latency I pattern is expressed dually in EBV and KSHV positive PEL cells, suggests that KSHV is the driving force behind the development of PEL (figure 1). ${ }^{12}$

Kaposi's sarcoma-associated herpes virusKSHV belongs to the $\gamma$-herpesvirus family, and is the first known human member of the genus Rhadinovirus. This virus infects endothelial/spindle cells and lymphocytes. ${ }^{20} 21$ In KSHV-associated cancers, viral presence has been demonstrated by immunohistochemistry and by PCR in tumour tissues, but not in the adjacent tissues ${ }^{22} 23$ providing the initial evidences in favour of its involvement in the oncogenic process. The methods used to detect KSHV differ in sensitivity and in target detection, and detect only limited aspects of the oncogenic role of $\mathrm{KSHV}^{24}$

\section{Cancers that have been attributed to KSHV}

A recent evaluation of the available epidemiological and molecular mechanistic evidence resulted in the classification of KSHV as a group 1 carcinogen. In the case of Kaposi's sarcoma (KS) and PEL the evidence supporting a causative role for KSHV was considered sufficient (table 1).

Table 2 Epstein-Barr virus latency pattern and associated malignancies

\begin{tabular}{lll}
\hline Latency type & $\begin{array}{l}\text { Viral genes } \\
\text { expressed }\end{array}$ & Associated malignancies \\
\hline Latency I & EBNA-1 & Burkitt's lymphoma \\
& EBERs & \\
BARF0 & \\
Latency II & EBNA-1 & Classical Hodgkin's lymphoma \\
& EBERs & Undifferentiated carcinoma \\
& LMP1 & Peripheral T/NK cell lymphoma \\
& LMP2 & \\
& BARF0 & \\
Latency III & All EBNAs & AIDS-associated lymphomas \\
& EBERs & Post-transplant lymphoproliferative disorders \\
& LMP1 & \\
& LMP2 & \\
& BARF0 & \\
\hline
\end{tabular}

NK cell, natural killer cells.
The epidemiological evidence supporting a causative role for KSHV in the pathogenesis of KS is strongly based on the fact that the virus is found in virtually all KS tumours, irrespective of the clinical form ('classic', African-endemic, HIV-associated, post-transplant $)^{2}$ (figure 3).

PEL is encountered in AIDS patients and transplant recipients. Owing to its rarity, the epidemiological argument in favour of a causative involvement of KSHV is limited to the fact that $\mathrm{KSHV}$ is consistently found in this lymphoma. However, there are the following biological evidences: the same latent viral proteins (LANA, vcyc, vFLIP, kaposin) found in KS are also expressed in PEL; in addition, one of the KSHV interferon regulatory factor homologues, VIRF-3, shows a latent gene expression pattern in lymphoma cells and there is also substantial expression of an interleukin 6 homologue, vIL-6. Finally, experimental data show that the continuous expression of these viral proteins is required for PEL cell survival. ${ }^{11} 25-27$

\section{Human papillomavirus}

HPVs are small DNA viruses belonging to the family Papillomaviridae. $^{28} 29 \mathrm{HPV}$ have circular, double-stranded DNA genomes typically containing 8 genes; the L1 gene encodes the principal capsidic protein, and its nucleotide sequence constitutes the basis for HPV classification into more than 120 'types', ${ }^{28}$ while the L2protein is a minor virion component. ${ }^{30} 31$ The different HPV types are generally divided into those preferentially infecting the skin versus mucosal surfaces, the infection of the latter being associated with neoplastic diseases of the anogenital tract, and of head and neck squamous cell carcinomas. ${ }^{29} 3233$ Among the genital HPVs, 15 types are considered 'high-risk' (in particular, HPV 16 and 18 that are responsible of $>70 \%$ of the cancers), with over $99 \%$ of cervical lesions containing viral sequences. ${ }^{34}$

HPV infection requires that the virus passes through the epithelial basal layer and enters the basal cells, but the mode of entry is still debated. ${ }^{30-32}$ While many HPV types produce only productive infection, oncogenic HPV types contribute to the

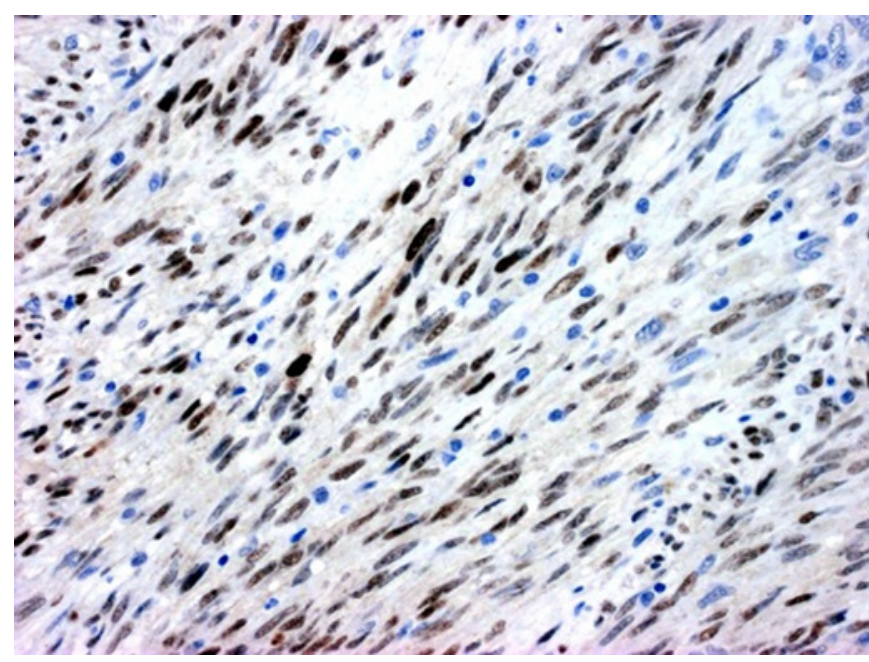

Figure 3 KSHV-associated Kaposi sarcoma (KS). Neoplastic spindle cells from a tumourous phase Kaposi's sarcoma display nuclear positivity KSHV with a speckled pattern of staining. KSHV positivity assessed by immunohistochemistry for ORF73/KSHV in formalin-fixed paraffin-embedded tissue section. Haematoxylin counterstain, original magnification $\times 40$. KSHV, Kaposi's sarcoma associated herpes virus; $\mathrm{ORF}$, open reading fragment. 
development of cancers, often arising at sites where productive infection cannot be supported. ${ }^{30}$

In these 'high-risk' HPV types, the E5, E6 and E7 genes are responsible for the production of the viral oncoproteins involved in the initiation and progression of cervical cancer.

\section{Cancers that have been attributed to HPV}

HPV infection-attributable cancers include carcinoma of the cervix, vulva, vagina, penis, anus, oral cavity and oropharynx and tonsil. ${ }^{11}$ The oncogenic types found most frequently in cervical cancer (HPV-16, 18, 31, 33, 35, 45, 52, 58) and four types less constantly found (HPV-39, 51, 56, 59) were classified in group 1 by the recent IARC evaluation. ${ }^{2}$ Of these, HPV-16 and 18 carry, by far, the highest risk of cancer. ${ }^{35-40}$ Several comprehensive studies have confirmed that these 'high-risk' HPV types cause virtually all cases of cervical cancer worldwide. ${ }^{39} 40$ HPV-16 is also the most important cause of anal cancer ${ }^{2}$ (figure 4).

Epidemiological data provide clues to the role of immune suppression in the development of HPV-related cancers on HIV setting. ${ }^{41}$ Yet, cervical cancer does not appear to have decreased after the introduction of highly active antiretroviral therapy. ${ }^{42} 43$

\section{PATHOLOGICAL AND VIROLOGICAL TECHNIQUES: CLINICAL APPLICATIONS EBV infection}

The most abundant viral transcripts in latently infected cells, EBER1 and EBER2 (collectively called EBER), function to inhibit interferon-mediated antiviral effects and apoptosis. These two transcripts are expressed at around one million copies per latently infected cells. They are considered to be the best natural markers of latent infection. In situ hybridisation targeting one or both EBERs is the gold standard assay for reliably detecting EBV infection on formalin- and Bouin-fixed tumour samples. ${ }^{44}$ Today, this is a conventional technique to diagnose and classify NPCand EBV-associated lymphomas in non-immunosuppressed

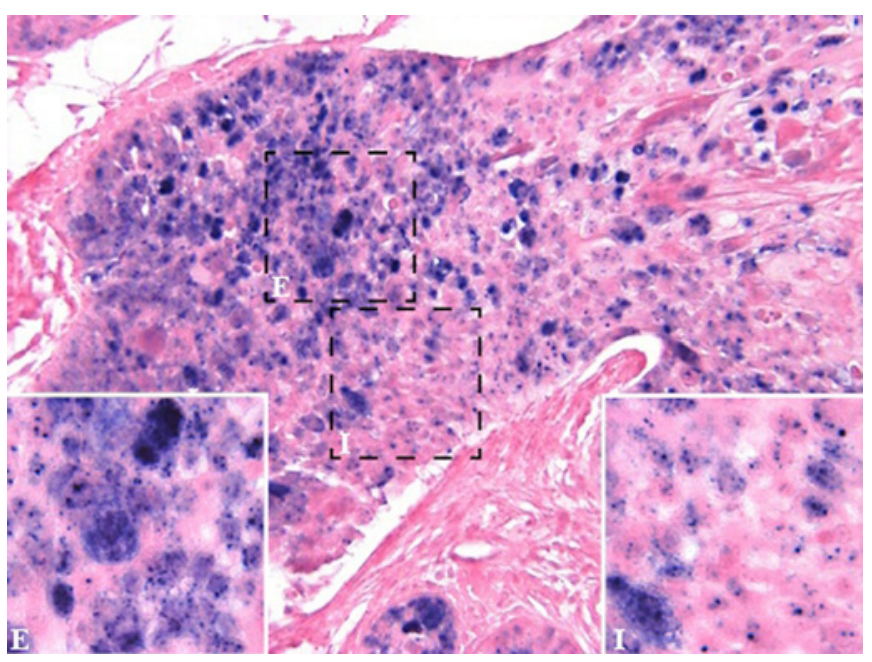

Figure 4 Human papillomavirus (HPV)-associated anal carcinoma. The figure shows an anal carcinoma in which neoplastic cells present "highrisk' HPV infection either in episomal (E) or integrated (I) form. The positivity is nuclear. Inset: High magnification showing episomal (E) and integrated (I) pattern of staining. HPV positivity assessed by in situ hybridisation using a cocktail of probes recognising HR HPV types 16, $18,31,33,35,39,45,51,52,56,58,59,68$ and 70 in formalin-fixed paraffin-embedded section. Fast red counterstain, original magnification $\times 40, \times 60$ (inset). hosts, and in AIDS-associated lymphomas and post-transplant lymphoid disorders. ${ }^{45}$

To identify the pattern of EBV latency expressed by tumour cells, the expression of EBV latent genes can be assessed by immunohistochemistry on fixed tissue samples by analysing EBV LMP1, LMP2a and EBV-encoded nuclear antigen 2.

The viral prevalence rates in tumours by using the various molecular methods are quite variable. ${ }^{46}$ Some studies employed PCR to amplify conserved regions of EBV DNA, but with this technique, false negative results may depend on the fact that PCR detects few genes at one time, while false positive results may be attributed to infiltrating lymphocytes, thus not allowing the distinction between lesion-specific EBV and background infection. ${ }^{47-50}$

Recent studies suggest that EBV genome chips covering the entire viral genome can optimally detect EBV infection in tumour cells ${ }^{46}$; EBV chips have the advantage of a low false positive rate and the simultaneous analysis of genome-wide viral gene expression pattern in tumour tissues. By using EBV chips, Yen et $a l^{51}$ have demonstrated an increased sensitivity of EBV detection rate in oral cancer cells as compared with traditional molecular assays (from $15-77 \%$ to $82 \cdot 5 \%$ ).

Molecular methods have also demonstrated the monoclonal nature of EBV infection in EBV-related cancers. ${ }^{45}$

\section{EBV DNA viraemia}

Over the last few years, EBV DNA viral load testing has been extensively implemented for the prediction, diagnosis and monitoring of EBV-associated diseases. ${ }^{47} 48 \quad 52 \quad 53$ EBV DNA normally resides inside circulating $B$ lymphocytes, whereas patients with EBV-associated cancers also have detectable viral DNA in plasma, making the detection of extracellular viral load a suitable specimen type for clinical purposes. ${ }^{48} 53$

In EBV-related cancers, viral load may be produced through different mechanisms and have peculiar significance in cancers of different origin. At least two different mechanisms may be hypothesised. In the first, including cancers arising in immunocompetent hosts (NPC, HL, NK/T cell lymphomas), the EBVDNA detectable in the peripheral blood is not related to viral reactivation, but rather, derives directly from the necrosis/ apoptosis of tumour cells. ${ }^{54} 55$ For this reason, it may be considered as a real tumour biomarker.

In areas where NPC is frequent, anti-viral capsidic antigen $\operatorname{Ig} \mathrm{A}$ antibody test is used to screen populations at higher risk to develop the tumour. Serological assays are cheap and technically easy, but suffer from limited specificity. For this reason, EBV DNA has been proposed as a confirmatory test, when immunoglobulin A-viral capsidic antigen results are positive or indeterminate. ${ }^{56}$ EBV DNA assay may be also used as a diagnostic tool, for example, when histology gives inconclusive results. In high-incidence areas, plasma EBV DNA has a diagnostic sensitivity of $95 \%,{ }^{56}$ while in low-incidence regions, sensitivity is reduced to $70-75 \% .{ }^{51} 57$ Some authors tried to support the usefulness of EBV DNA determination in nasopharyngeal brushings, rather than in the peripheral blood. ${ }^{58}$

Pre-therapy EBV DNA is an independent prognostic factor to international union against cancer staging in NPC. ${ }^{59}$ In fact, several studies have demonstrated that overall survival, or relapse-free survival, were statistically significantly lower in NPC patients with elevated EBV DNA. ${ }^{59} 60$ In order to identify prognostic factors related to therapy, Lo et al demonstrated that EBV DNA declined after radiotherapy ${ }^{49}$; however, plasma EBV DNA levels depend on the balance between production and 
catabolism, and limited information is available at the moment on these issues. In agreement with histopathological data, EBV DNA is detectable in all EBV positive HL before therapy, ${ }^{61}{ }^{62}$ and in one study predicts response to therapy, ${ }^{62}$ but the use of EBV DNA as a diagnostic or predictive marker in HL patients is not recommended.

In the second type of cancers, including EBV-related lymphoproliferative disorders, EBV DNA is actively released from circulating EBV-positive $B$ cells proliferating because of the absence of an effective T-cell control. ${ }^{63}$ In this case, EBV DNA level may not be considered as a true tumour marker, but rather a measure of immunosuppression. Having in mind this limitation, EBV viral load is considered as very useful to predict, diagnose and monitor post-transplant lymphoproliferative disorders. ${ }^{48}$

Approximately $20 \%$ of HIV positive asymptomatic patients have a detectable viral load in the peripheral blood, and it has been hypothesised that EBV DNA may be used as a reliable predictor of the development of non-HLs, but detailed longitudinal studies failed to support this hypothesis. ${ }^{64} 65$

The methods currently used to measure EBV DNA consistently differ for the EBV genome regions detected, for the detection techniques used and for the type of external standards used to set up the standard curve, producing a significant variability among laboratories. ${ }^{47} 53$ Despite these limitations, EBV viral load is considered relevant for the management of EBV-associated cancers.

\section{KSHV infection}

The detection of KSHV infection in tumour cells is the prerequisite for a precise diagnosis of KS or PEL. KSHV infection can be assessed on formalin-fixed cytospin (or cell blocks) obtained from effusions or formalin-fixed tissue sections. ${ }^{12} 66$

The presence of KSHV can be ascertained by immunohistochemistry on cells or sections using antibodies against the KSHV latency-associated nuclear antigen encoded by viral open reading fragment (ORF) 73. As KSHV in tumour cells is in latent phase, ORF73 immunohistochemistry is the gold standard to reliably detect KSHV infection in tumour samples. ${ }^{12}$ This antigen is detectable in the nucleus of almost all the infected cells. Positive cases can also be tested for viral interleukin-6 (vIL-6), a KSHV cytokine homologue. Staining for vIL-6 is cytoplasmic and is restricted to a limited number of infected cells. Positive controls for ORF73 consist of KS biopsy samples, whereas positive controls for vIL-6 consist of AIDS-associated multicentric Castleman's disease (MCD) biopsy samples. ${ }^{12}$

The presence of KSHV can be confirmed by PCR analysis of three KSHV regions (ie, K330233, ORFK9-3, ORF72) using primer sequences. ${ }^{66}$

\section{KSHV viraemia}

During KSHV infection, viral DNA can be detected both in peripheral blood mononuclear cells and as cell-free DNA (plasma viraemia $)^{67}$ and may be used to predict, diagnose or prognosticate diseases associated with this virus.

Engels et al have demonstrated that, among individuals with HIV-KS co-infection, KSHV viraemia predicts the further development of $\mathrm{KS}^{68}{ }^{69}$ while no conclusive studies are presently available on the predictive role of viraemia in the development of KS in the HIV-negative population. ${ }^{70}$

There is a wide consensus in the scientific literature that KSHV viraemia does not have a diagnostic role in any of the KSHV-associated diseases, ${ }^{71}$ while several studies have considered KSHV viraemia as a useful prognostic indicator of disease progression and/or shorter survival in $\mathrm{KS}^{70} 72$ and PEL. ${ }^{73}$
Finally, KSHV viraemia has been demonstrated to be a reliable method to monitor the response of KS to highly active antiretroviral therapy ${ }^{67} 74$ or to anti-tumour chemotherapy in $\mathrm{MCD}^{75} 76$

\section{HPV infection}

In developed countries, a cervical Papanicolaou (Pap) test (a screening test to detect cervical cell changes) is still used to detect abnormal cells that may develop into cancer. On the other hand, genital warts that are the only visible sign of 'low-risk' HPV continue to be identified with a visual check.

An HPV test for cervical infection as a follow-up for women who have an ambiguous Pap test for general cervical cancer screening has recently been adopted. This HPV test (HC2) can identify 'low' and 'high-risk' types of HPV associated with the development of cervical cancer. However, today it is possible to test for HPV DNA in extracervical infections by means of multiple approaches. Many assays for HPV detection are available today, each characterised by its own analytical sensitivity. In situ hybridisation can also be applied to detect HPV in slides (scrapes or tissue sections). There are two commercially available systems able to detect HPV in tissue samples, that is, the Ventana System (Ventana Systems, Inc., Harvard, MA, USA), able to identify 'high-risk' HPV types, whereas Dako Probe (Dako, Denmark) is specific for detecting HPV16. Both methods allow the identification of the integrated and episomal form. P16 immunohistochemistry has been advocated as a surrogate marker of 'high-risk' HPV infection. Finally, there are the PCRbased assay systems, often linked to a specific genotyping system.

With the presently available assays that have extremely high analytical sensitivities, there is the risk that the test is too sensitive and detects virus without underlying biological or clinical consequences. There is sufficient evidence that increased analytical sensitivities of assays result in decreased clinical specificities (ie, more positive test results in the absence of virusinduced disease). Thus, a positive 'high-risk' HPV test does not automatically mean that the virus has been involved in the initiation or maintenance of carcinogenesis. ${ }^{77}$

HPV DNA may be present in cervical lesions in the absence of any overt disease, and may represent a silent viral infection, possibly because of an efficient immune control at the mucosal surface.

Since HPV DNA integration is found in most invasive cancers, it has been considered as a step promoting viral carcinogenesis; however, experimental data are conflicting, and this topic is still debated. $^{78} 79$ The quantitative measurement of HPV DNA integration in human cells may offer information to classify HPV-related cancers based on molecular characteristics, but the significance of quantitative data is still debated, since viral load values are an average summed over many infected and uninfected cells. $^{78}$

Owing to the fact that viral integration disrupts the E2 gene, while the E6 gene is maintained, the E2/E6 ratio accurately measures the amount of integrated HPV in human tumour samples. ${ }^{80} \mathrm{HPV}$ E6/E7 mRNA testing may also be a potentially useful biomarker to evaluate the risk of progression of HPV-related pre-neoplastic lesions. ${ }^{81}$

Since HPV-associated cancers are associated with 'high-risk' viral types, HPV typing is essential for diagnostic and research purposes. Typing can be performed by amplifying HPV L1 gene combined with reverse line blotting assay of sequencing. ${ }^{78}$

Several studies suggest that in a proportion of HPV-related lesions, several viral types are simultaneously detected ${ }^{78} 81$ 
Table 3 Biological agents assessed by the International Agency for Research on Cancer monograph working group (adapted from Bouvard et $a l^{2}$ )

\begin{tabular}{ll}
\hline Group 1 agent & $\begin{array}{l}\text { Other sites with limited } \\
\text { evidence in humans }\end{array}$ \\
\hline Epstein-Barr virus (EBV) & $\begin{array}{l}\text { Gastric carcinoma, } \\
\text { lymphoepithelioma-like carcinoma }\end{array}$ \\
Kaposi sarcoma herpesvirus (KSHV) & $\begin{array}{l}\text { Multicentric castleman's disease } \\
\text { Cancer of the larynx }\end{array}$ \\
\hline
\end{tabular}

However, there is a lack of consensus in literature about the prevalence and carcinogenic role of co-infections with multiple HPV types in cervical and head and neck carcinomas. ${ }^{82}$

In conclusion, virological assays to detect HPV may measure the qualitative and quantitative presence of HPV DNA and determine the HPV-infecting type.

In the future, molecular assays on laser-microdissected single tumour cells may be essential to study the viral oncogenic pathways and to obtain more reliable diagnostic and prognostic information on HPV-related cancers.

\section{FUTURE SCENARIO}

Owing to the fact that a fraction of malignancies can be caused by chronic infections, vaccination may be effective against the onset of these cancers. The future scenario we may have to face ahead of us in case the development of vaccines were feasible, and vaccination campaigns were effective, may consist of a $15-20 \%$ reduction of human malignant tumours worldwide.

In conclusion, the future scenario is promising. Yet, several issues relating to viral agents and their role on carcinogenesis are still open, and tumour prevention and treatment. There is still much to do in these fields as far as research is concerned. First, we need to investigate whether some neoplastic diseases with

\section{Interactive multiple choice questions}

This JCP review article has an accompanying set of multiple choice questions (MCOs). To access the questions, click on BMJ Learning: Take this module on BMJ Learning from the content box at the top right and bottom left of the online article. For more information please go to: http://jcp.bmj.com/education. Please note: The MCOs are hosted on BMJ Learning - the best available learning website for medical professionals from the BMJ Group. If prompted, subscribers must sign into JCP with their journal's username and password. All users must also complete a onetime registration on BMJ Learning and subsequently log in (with a BMJ Learning username and password) on every visit.

\section{Take home messages}

Infectious agents are implicated as causative factors in a significant proportion of human cancers. Due to their direct carcinogenic role, EBV, KSHV, and HPV are particularly important among viruses. Therefore the use of well-established pathological and virologic techniques is helpful to diagnose and characterize viral-associated cancers. However, several issues relating to viral related cancers are still open including tumour prevention and treatment. limited evidence (table 3) are only coincidentally bound to such biological agents. We are referring to carcinoma of the larynx and $\mathrm{HPV}$, carcinoma of the stomach and EBV, MCD and KSHV. Moreover, other biological agents are still under investigation to establish whether they belong to group 1, that is, to cancercausative biological agents. Therefore, the fight against cancer both in developing and developed countries can benefit from this knowledge, specifically of the induction of complete protection against primary infection, or prevention of chronic infections and of the disease. It is still doubtful whether which of the two is the most effective/feasible way.

Contributors $\mathrm{AC}$ and PDP contributed equally to this work.

Competing interests None

Provenance and peer review Not commissioned; externally peer reviewed.

\section{REFERENCES}

1. World Cancer Report. In: Boyle P, Levin B, eds. World Health Organization, International Agency for Research on Cancer. World Health Organization International Agency for Research on Cancer, Lyon, France, 2008.

2. Bouvard V, Baan R, Straif K, et al. A review of human carcinogens. Part B: biological agents. Lancet Oncol 2009;10:321-2.

3. Young LS, Rickinson AB. Epstein-Barr virus: 40 years on. Nat Rev Cancer 2004:4:757-68.

4. Cohen JI. Epstein-Barr virus infection. N Engl J Med 2000;343:481-92.

5. Kutok JL, Wang F. Spectrum of Epstein-Barr virus-associated diseases. Annu Rev Pathol Mech Dis 2006;1:375-404.

6. Thompson MP, Kurzrock R. Epstein-Barr virus and cancer. Clin Cancer Res 2004:10:803-21.

7. Young LS, Murray PG. Epstein-Barr virus and oncogenesis: from latent genes to tumours. Oncogene 2003:22:5108-21.

8. World Health Organization classification of tumours. Pathology and genetics In: Barnes L, Eveson JW, Reichart P, et al, eds. Head \& Neck Tumours. Lyon: IARC Press, 2005.

9. IARC Monographs On The Evaluation Of Carcinogenic Risks To Humans, Volume 70 (1997), Epstein-Barr Virus and Kaposi's Sarcoma Herpesvirus/Human Herpesvirus 8 Lyon, France, 1997.

10. Sousa H, Pinto-Correia AL, Medeiros R, et al. Epstein-Barr virus is associated with gastric carcinoma: the question is what is the significance? World $\mathrm{J}$ Gastroenterol 2008:14:4347-51.

11. Carbone A, Gloghini A, Dotti G. EBV-associated lymphoproliferative disorders: classification and treatment. Oncologist 2008;13:577-85.

12. Carbone A, Cesarman E, Spina M, et al. HIV-associated lymphomas and gamma-herpesviruses. Blood 2009;113:1213-24

13. Carbone A, Tirelli U, Gloghini A, et al. Human immunodeficiency virus associated systemic lymphomas may be subdivided into two main groups according to Epstein-Barr viral latent gene expression. J Clin Oncol 1993;11:1674-81.

14. Hamilton-Dutoit SJ, Rea D, Raphael M et al. Epstein-Barr virus-latent gene expression and tumor cell phenotype in acquired immunodeficiency syndrome-related non-Hodgkin's lymphoma. Correlation of lymphoma phenotype with three distinct patterns of viral latency. Am J Pathol 1993;143:1072-85.

15. Klein E, Kis LL, Klein G. Epstein-Barr virus infection in humans: from harmless to life endangering virus-lymphocyte interactions. Oncogene 2007;26:1297-305.

16. Biggar RJ, Jaffe ES, Goedert JJ, et al. Hodgkin lymphoma and immunodeficiency in persons with HIV/AIDS. Blood 2006;108:3786-91.

17. Grogg KL, Miller RF, Dogan A. HIV infection and lymphoma. J Clin Pathol 2007;60:1365-72.

18. Tirelli U, Errante D, Dolcetti $\mathrm{R}$, et al. Hodgkin's disease and human immunodeficiency virus infection: clinicopathologic and virologic features of 114 patients from the Italian cooperative group on AIDS and tumors. J Clin Oncol 1995;13:1758-67.

19. Carbone A, Gloghini A, Larocca LM, et al. Human immunodeficiency virusassociated Hodgkin's disease derives from post-germinal center B cells. Blood 1999:93:2319-26.

20. Schulz TF. The pleiotropic effects of Kaposi's sarcoma herpesvirus. J Pathol 2006;208:187-98.

21. Hengge UR, Ruzicka T, Tyring SK, et al. Update on kaposi's sarcoma and other HHV8 associated diseases. Part 2: pathogenesis, Castleman's disease, and pleural effusion lymphoma. Lancet Infect Dis 2002;2:344-52.

22. Kalt I, Masa SR, Sarid R. Linking the Kaposi's sarcoma-associated herpesvirus (KSHV/HHV-8) to human malignancies. Methods Mol Biol 2009;471:387-407.

23. Pak F, Pyakural P, Kokhaei $\mathrm{P}$, et al. HHV-8/KSHV during the development of kaposi's sarcoma: evaluation by polymerase chain reaction and immunohistochemistry. J Cutan Pathol 2005;32:21-7.

24. Stürzl M, Konrad A, Alkharsah KR, et al. The contribution of systems biology and reverse genetics to the understanding of Kaposi's sarcoma-associated herpesvirus pathogenesis in endothelial cells. Thromb Haemost 2009;102:1117-34. 
25. Godfrey A, Anderson J, Papanastasiou A, et al. Inhibiting primary effusion lymphoma by lentiviral vectors encoding short hairpin RNA. Blood 2005; 105:2510-18.

26. Guasparri I, Keller SA, Cesarman E. KSHV vFLIP is essential for the survival of infected lymphoma cells. J Exp Med 2004;199:993-1003.

27. Wies $\mathbf{E}$, Mori $Y$, Hahn $A$, et al. The viral interferon-regulatory factor-3 is required for the survival of KSHV-infected primary effusion lymphoma cells. Blood 2008;111:320-7.

28. Bernard HU, Burk RD, Chen Z, et al. Classification of papillomaviruses (PVs) based on 189 PV types and proposal of taxonomic amendments. Virology 2010;401:70-9.

29. Zur Hausen H. Papillomaviruses and cancer: from basic studies to clinical application. Nat Rev Cancer 2002;2:342-50.

30. Doorbar J. Molecular biology of human papillomavirus infection and cervical cancer Clin Sci (Lond) 2006;110:525-41.

31. Moody CA, Laimins LA. Human papillomavirus oncoproteins: pathways to transformation. Nat Rev Cancer 2010;10:550-60.

32. Hebner CM, Laimins LA. Human papillomaviruses: basic mechanisms of pathogenesis and oncogenicity. Rev Med Virol 2006;16:83-97.

33. Gillespie MB, Rubinchik S, Hoel B, et al. Human papillomavirus and oropharyngeal cancer: what you need to know in 2009. Curr Treat Options Oncol 2009;10:296-307

34. Walboomers JM, Jacobs MV, Manos MM, et al. Human papillomavirus is a necessary cause of invasive cervical cancer worldwide. J Pathol 1999;189:12-19.

35. Chung $\mathbf{C H}$, Gillison ML. Human papillomavirus in head and neck cancer: its role in pathogenesis and clinical implications. Clin Cancer Res 2009;15:6758-62.

36. de Villiers EM, Fauquet C, Broker TR, et al. Classification of papillomaviruses. Virology 2004;324:17-27.

37. IARC. Human papillomaviruses. IARC Monogr Eval Carcinog Risks Hum 2007; 90:1-636

38. IARC. Monograph on the Evaluation of Carcionogenic Risks to Humans. vol. 64 WHO, Lyon, France, 1995.

39. Bosch FX, Burchell AN, Schiffman M, et al. Epidemiology and natural history of human papillomavirus infections and type-specific implications in cervical neoplasia. Vaccine 2008:26(Suppl 10):K1-16

40. Smith JS, Lindsay L, Hoots B, et al. Human papillomavirus type distribution in invasive cervical cancer and high-grade cervical lesions: a meta-analysis update. Int J Cancer 2007;121:621-32.

41. Frisch M, Biggar RJ, Goedert JJ. Human papillomavirus-associated cancers in patients with human immunodeficiency virus infection and acquired immunodeficiency syndrome. J Natl Cancer Inst 2000;92:1500-10.

42. Engels EA, Pfeiffer RM, Goedert JJ, et al. Trends in cancer risk among people with AIDS in the United States 1980-2002. AIDS 2006;20:1645-54.

43. Biggar RJ, Chaturvedi AK, Goedert JJ, et al. AIDS-related cancer and severity of immunosuppression in persons with AIDS. J Natl Cancer Inst 2007;99:962-72.

44. Schulz TF. Cancer and viral infections in immunocompromised individuals. Int $J$ Cancer 2009;125:1755-63.

45. Gulley ML, Tang W. Laboratory assays for Epstein-Barr virus-related disease. J Mol Diagn 2008;10:279-92.

46. Li C, Chen RS, Hung SK, et al. Detection of Epstein-Barr virus infection and gene expression in human tumors by microarray analysis. J Virol Methods 2006;133:158-66

47. De Paoli P, Pratesi C, Bortolin MT. The Epstein Barr virus DNA levels as a tumor marker in EBV-associated cancers. J Cancer Res Clin Oncol 2007;133:809-15.

48. Gulley ML, Tang W. Using Epstein-Barr viral load assays to diagnose, monitor, and prevent posttransplant lymphoproliferative disorder. Clin Microbiol Rev 2010:23:350-66.

49. Lo YM, Leung SF, Chan LY, et al. Kinetics of plasma Epstein-Barr virus DNA during radiation therapy for nasopharyngeal carcinoma. Cancer Res 2000;60:2351-5.

50. Gulley ML. Molecular diagnosis of Epstein-Barr virus-related diseases. J Mol Diagn 2001;3:1-10.

51. Yen CY, Lu MC, Tzeng CC, et al. Detection of EBV infection and gene expression in oral cancer from patients in Taiwan by microarray analysis. J Biomed Biotechnol 2009;2009:904589.

52. Tsai DE, Douglas L, Andreadis C, et al. EBV PCR in the diagnosis and monitoring of posttransplant lymphoproliferative disorder: results of a two-arm prospective trial. Am J Transp/ 2008;8:1016-24.

53. Gärtner B, Preiksaitis JK. EBV viral load detection in clinical virology. J Clin Virol 2010;48:82-90

54. To EW, Chan KC, Leung SF, et al. Rapid clearance of plasma Epstein-Barr virus DNA after surgical treatment of nasopharyngeal carcinoma. Clin Cancer Res 2003;9:3254-9.

55. Shotelersuk K, Khorprasert C, Sakdikul S, et al. Epstein-Barr virus DNA in serum/ plasma as a tumor marker for nasopharyngeal cancer. Clin Cancer Res 2000;6:1046-51.

56. Leung SF, Tam JS, Chan AT, et al. Improved accuracy of detection of nasopharyngeal carcinoma by combined application of circulating Epstein-Barr virus DNA and anti-Epstein-Barr viral capsid antigen IgA antibody. Clin Chem 2004;50:339-45.
57. Bortolin MT, Pratesi C, Dolcetti R, et al. Clinical value of Epstein-Barr virus DNA levels in peripheral blood samples of Italian patients with undifferentiated carcinoma of nasopharyngeal type. Cancer Lett 2006;233:247-54.

58. Tong JH, Tsang RK, Lo KW, et al. Quantitative Epstein-Barr virus DNA analysis and detection of gene promoter hypermethylation in nasopharyngeal (NP) brushing samples from patients with NP carcinoma. Clin Cancer Res 2002:8:2612-19.

59. Leung SF, Zee B, Ma BB, et al. Plasma Epstein-Barr viral deoxyribonucleic acid quantitation complements tumor-node-metastasis staging prognostication in nasopharyngeal carcinoma. J Clin Oncol 2006:24:5414-18.

60. Leung SF, Chan AT, Zee B, et al. Pretherapy quantitative measurement of circulating Epstein-Barr virus DNA is predictive of posttherapy distant failure in patients with early-stage nasopharyngeal carcinoma of undifferentiated type. Cancer 2003;98:288-91

61. Gallagher A, Armstrong AA, MacKenzie J, et al. Detection of Epstein-Barr virus (EBV) genomes in the serum of patients with EBV-associated Hodgkin's disease. Int $J$ Cancer 1999;84:442-8.

62. Gandhi MK, Lambley E, Burrows J, et al. Plasma Epstein-Barr virus (EBV) DNA is a biomarker for EBV-positive hodgkin's lymphoma. Clin Cancer Res 2006;12:460-4.

63. Taylor AL, Marcus R, Bradley JA. Post-transplant lymphoproliferative disorders (PTLD) after solid organ transplantation. Crit Rev Oncol Hematol 2005;56:155-67.

64. Fan So, Ma J, Zhou J, et al. Differential expression of Epstein-Barr virus-encoded RNA and several tumor-related genes in various types of nasopharyngeal epithelia lesions and nasopharyngeal carcinoma using tissue microarray analysis. Hum Patho 2006;37:593-605.

65. Van Baarle D, Wolthers KC, Hovenkamp E, et al. Absolute level of Epstein-Barr virus DNA in human immunodeficiency virus type 1 infection is not predictive of AIDS related non-hodgkin lymphoma. J Infect Dis 2002;186:405-9.

66. Carbone A, Gloghini A, Vaccher E, et al. Kaposi's sarcoma-associated herpesvirus/ human herpesvirus type 8-positive solid lymphomas: a tissue-based variant of primary effusion lymphoma. J Mol Diagn 2005:7:17-27.

67. Tedeschi R, Marus A, Bidoli $E$, et al. Human herpesvirus 8 DNA quantification in matched plasma and PBMCs samples of patients with HHV8-related lymphoproliferative diseases. J Clin Virol 2008;43:255-9.

68. Engels EA, Biggar RJ, Marshall VA, et al. Detection and quantification of kaposi's sarcoma-associated herpesvirus to predict AIDS-associated kaposi's sarcoma. AIDS 2003;17:1847-51.

69. Whitby D, Howard MR, Tenant-Flowers M, et al. Detection of kaposi sarcoma associated herpesvirus in peripheral blood of HIV-infected individuals and progression to kaposi's sarcoma. Lancet 1995;346:799-802.

70. Pellet C, Kerob D, Dupuy A, et al. Kaposi's sarcoma-associated herpesvirus viremia is associated with the progression of classic and endemic kaposi's sarcoma. $J$ Invest Dermatol 2006;126:621-7.

71. Bower M, Collins S, Cottrill C, et al. British HIV Association guidelines for HIVassociated malignancies 2008. HIV Med 2008:9:336-88.

72. Quinlivan EB, Zhang C, Stewart PW, et al. Elevated virus loads of kaposi's sarcomaassociated human herpesvirus 8 predict Kaposi's sarcoma disease progression, but elevated levels of human immunodeficiency virus type 1 do not. $J$ Infect Dis 2002; 185:1736-44.

73. Simonelli C, Tedeschi R, Gloghini A et al. Prognostic factors in human herpesvirus 8-related lymphoproliferative disorders associated with HIV infection. J Clin Oncol 2006;24:209-10

74. Bourboulia D, Aldam D, Lagos D, et al. Short- and long-term effects of highly active antiretroviral therapy on kaposi sarcoma-associated herpesvirus immune responses and viraemia. AIDS 2004;18:485-93

75. Sullivan RJ, Pantanowitz L, Casper C, et al. HIV/AIDS: epidemiology, pathophysiology, and treatment of kaposi sarcoma-associated herpesvirus disease: kaposi sarcoma, primary effusion lymphoma, and multicentric castleman disease. Clin Infect Dis 2008; 47:1209-15

76. Oksenhendler E. HIV-associated multicentric castleman disease. Curr Opin HIV AIDS 2009:4:16-21.

77. Braakhuis BJ, Brakenhoff RH, Meijer CJ, et al. Human papilloma virus in head and neck cancer: the need for a standardised assay to assess the full clinical importance. Eur J Cancer 2009:45:2935-9.

78. Zaravinos A, Mammas IN, Sourvinos G, et al. Molecular detection methods of human papillomavirus (HPV). Int J Biol Markers 2009:24:215-22.

79. Briolat J, Dalstein V, Saunier M, et al. HPV prevalence, viral load and physical state of HPV-16 in cervical smears of patients with different grades of CIN. Int J Cance 2007:121:2198-204.

80. Klaes R, Woerner SM, Ridder R, et al. Detection of high-risk cervical intraepithelial neoplasia and cervical cancer by amplification of transcripts derived from integrated papillomavirus oncogenes. Cancer Res 1999:59:6132-6.

81. Cox JT. History of the use of HPV testing in cervical screening and in the management of abnormal cervical screening results. J Clin Virol 2009;45(Suppl 1): S3-12. Erratum in: J Clin Virol 2010:47:299.

82. Conway C, Chalkley R, High A, et al. Next-generation sequencing for simultaneous determination of HPV load, subtype, and associated genomic copy number changes in tumors. J Mol Diagn. Published Online First: 9 January 2012. PMID: 22240447 